\title{
A Comparison of the Black-Scholes Option Pricing Model and Its Alternatives
}

\author{
A B M Shahadat Hossain ${ }^{1}$, Maliha Tasmiah Noushin ${ }^{2}$ and Kamrul Hasan ${ }^{3}$ \\ ${ }^{1,3}$ Department of Applied Mathematics, Dhaka University, Dhaka-1000, Bangladesh \\ ${ }^{2}$ Institute of Natural Sciences, United International University (UIU), Dhaka-1212, Bangladesh
}

(Received: 14 February 2019; Accepted: 23 June 2019)

\begin{abstract}
In this paper we estimate European put option price by using awell-established option pricing model, namely, the Constant Elasticity of Variance (CEV) model for the elasticity parameter $\beta<2$ and then compare it with the benchmark BlackScholes (BS) model. We calculate the Greeks under the CEV model for $\beta=0,1$ and 1.95 and compare them with that of the B-S one. Finally, we investigate the put price and Greeks values for at-the-money (ATM), in-the-money (ITM) and outof-the-money (OTM) situations.
\end{abstract}

Keywords: European Put Options; Black-Scholes Model; Binomial Model; CEV Model.

\section{Introduction}

The Black-Scholes (BS) Model is the most gratified option pricing model for calculating the premium of an option. And it plays a very significant role on the way of traders price and hedge derivatives. There are five key determinants for calculating the price of an option namely stock price, strike price, volatility, maturity time, and short-term (risk free) interest rate. The BS model is used to evaluate the exact price of European call and put options for non-dividend paying stock during the options lifetime by using these determinants. Bonessand Samuelson first introduced that The Black Scholes (BS) model that assumes geometric Brownian motion with constant drift and volatility. This assumption was later adopted in the research of Black-Scholes and Merton ${ }^{1}$. Under these assumptions, the change in the asset price is log-normally distributed.

The further results on the CEV model for call option were estimated by Emanuel, D. C., \& MacBeth, J. D. ${ }^{2}$ and these results were published in the Journal of Financial and Quantitative Analysis. The CEV model accepts that volatility changes as the stock prices change. MacBeth and Merville ${ }^{4}$ compared the BS model against the CEV model based on this assumption. Blattberg and Gonedes suggest the volatility of the underlying asset is stochastic and random and Rosenberg suggests that volatility of the underlying stock follows an auto regressive scheme ${ }^{2}$. As the stock price increases, the volatility of the underlying stock decreases. This inverse behavior was founded by MacBeth and Merville ${ }^{3}$. Their results are compatible with the results of Geske. Beckers ${ }^{4}$ checked the assumption that the historical volatility is a function of the stock price. In this paper, we calculate the put option price and the Greeks under the $\mathrm{CEV}$ model with $\beta=0,1,1.95$ and compare these results with the BS one.

\section{Descriptions of the Models}

In this section, we will discuss the B-S and CEV option pricing models briefly that overviews the needs of work. For details, readers are referred ${ }^{5}$. Some common notations used for these models are as follows

*Author for correspondence. e-mail: abmsh@du.ac.bd $c:=$ European call option price

$p:=$ European put option price

$S_{0}:=$ Stock price at the initial stage

$X:=$ Strike price of the option

$\sigma:=$ Volatility of the option market

$r:=$ Continuously compounded risk-free rate

$T:=$ Time to maturity of the option

The BS Equation and Option Pricing Formula

The BS differential equation is

$$
\frac{\partial f}{\partial t}+r S \frac{\partial f}{\partial S}+\frac{1}{2} \sigma^{2} S^{2} \frac{\partial^{2} f}{\partial S^{2}}=r f
$$

And the BS formula for the prices of European put options is as follows

$$
p=X e^{-r T} N\left(-d_{2}\right)-S_{0} N\left(-d_{1}\right)
$$

where

$d_{1}=\frac{\ln \left(\frac{S_{o}}{X}\right)+\left(r+\frac{\sigma^{2}}{2}\right) T}{\sigma \sqrt{T}}$, and $d_{2}=d_{1}-\sigma \sqrt{T}$

The CEV Model

The CEV model proposes a relationship between the price of the stock and volatility as: $\sigma(S, t)=\delta S^{\frac{\beta}{2}-1}$, where $\delta, \beta \in R$ and the elasticity of return variance w.r.to stock price equals $\beta-2$. Note that volatility and price are inversely related when $\beta<2$. The stock price is followed by thefollwoing diffusion process

$$
d S=(r-q) S d t+\delta S^{\beta} / 2 d Z
$$

where $d Z$ is a Weiner process.

Also note that if $\beta=2$ (i.e., the elasticity is zero), logarithm of stock prices is normally distributed and thevariance is constant which is expected in the BS model. In case of a perfect hedging configured between a European option and its underlying stock (discussed in Cox and Ross $^{6}$ ) and risk neutral environment, we can value an option 
by calculating the discounted expected value of its payoff. The CEV diffusion process described above by equation (3) layers the log- normal assumption of Black and Scholes (1973) and Merton (1973) $(\beta=2)$, as well as the absolute diffusion $(\beta=0)$ and the square-root diffusion $(\beta=1)$ models of Cox and Ross (1976), as special cases. Cox displays that, for a CEV process with, $<2$, the density function of $S_{T}$, the stock price at maturity, $T$, conditional on $S(t<T)$, the current stock price, in a risk- neutral world is

$$
\begin{gathered}
f\left(S_{T}, T ; S_{t}, t\right)=(2-\beta) k^{\left(\frac{1}{2-\beta}\right)}\left(x \omega^{1-2 \beta}\right)^{\frac{1}{4-2 \beta}} \\
\exp (-x-w) I_{\frac{1}{2-\beta}}(2 \sqrt{x \omega}),
\end{gathered}
$$

where

$$
\begin{gathered}
\tau=T-t, \\
k=\frac{2(r-q)}{\delta^{2}(2-\beta)[\exp ((r-q)(2-\beta) \tau)-1]} \\
x=k S_{t}^{(2-\beta)} \exp ((r-q)(2-\beta) \tau) \\
\omega=k S_{t}^{(2-\beta)}
\end{gathered}
$$

$I_{q}($.$) denotes the modified Bessel function of the first kind$ of order $q$ and $r$ is the risk-less interest rate.

Thus, the time-t value of a European-style put for $\beta<2$ is given by

$$
\begin{aligned}
& p=X \exp (-r \tau) \quad Q\left(2 x ; \frac{2}{2-\beta}, 2 y\right) \\
& -S_{t} \exp (-q \tau) \quad\left[1-Q\left(2 y ; 2+\frac{2}{2-\beta}, 2 x\right)\right] \\
& \text { or }, p=X \exp (-r \tau) \quad\left(1-F\left(2 x ; \frac{2}{2-\beta}, 2 y\right)\right) \\
& -S_{t} \exp (-q \tau) \quad F\left(2 y ; 2+\frac{2}{2-\beta}, 2 x\right) \\
& \text { or, } \quad p=X \exp (-r \tau)\left[1-\chi^{2}(c ; b, a)\right] \\
& -S_{t} \exp (-q \tau) \chi^{2}(a ; b+2, c)
\end{aligned}
$$

with $Q(w ; v, \lambda)$ denotes the non-central Chi square complementary distribution function withdegrees of freedom $v$ and non-centrality parameter $\lambda$ and where

$$
\begin{aligned}
& k=\frac{2(r-q)}{\delta^{2}(2-\beta)[\exp ((r-q)(2-\beta) \tau)-1]} \\
& 2 x=2 k S_{t}^{(2-\beta)} \exp ((r-q)(2-\beta) \tau)=c \\
& 2 y=2 k X^{2-\beta}=a \\
& \delta=\sigma S^{1-\frac{\beta}{2}} \\
& b=2 /(2-\beta), \text { for } \beta<2 .
\end{aligned}
$$

\section{Results and Discussion}

In this section, we will focus our analysis on European put options. The BS model equations (2) gives the European put price results while the equation (5) provides European option price under the $\mathrm{CEV}$ model for $\beta<2$.

The following table presents European put option prices under the $\mathrm{BS}$ and $\mathrm{CEV}$ assumption for different specifications of the option parameters. Parameters used in the calculations are

$S_{o}=30, X=20,25,30,35,40 ; r=0.05$;

$\sigma=0.2,0.25,0.30 ; q=0, \tau=0.5,1$

Table 1. BS and CEV European put price with $\beta=$ 1. 95, 1, 0and $\sigma=0.2,0.25$, and 0.30

\begin{tabular}{|c|l|l|l|l|l|}
\hline$\sigma$ & $\mathrm{X}$ & $\begin{array}{l}\text { BS } \\
\text { Price }\end{array}$ & $\begin{array}{l}\text { CEV } \\
(\beta=1.95)\end{array}$ & $\begin{array}{l}\text { CEV } \\
(\beta=1)\end{array}$ & $\begin{array}{l}\text { CEV } \\
(\beta=0)\end{array}$ \\
\hline \multirow{5}{*}{$\mathbf{0 . 2 0}$} & 20 & 0.0186 & 0.0193 & 0.0378 & 0.0689 \\
\cline { 2 - 6 } & 25 & 0.3229 & 0.3255 & 0.3748 & 0.4323 \\
\cline { 2 - 6 } & 30 & 1.6721 & 1.6721 & 1.6730 & 1.6761 \\
\cline { 2 - 6 } & 35 & 4.5001 & 4.4959 & 4.4184 & 4.3417 \\
\cline { 2 - 6 } & 40 & 8.4361 & 8.4313 & 8.3487 & 8.2758 \\
\hline \multirow{5}{*}{$\mathbf{0 . 2 5}$} & 20 & 0.0802 & 0.0825 & 0.1358 & 0.2144 \\
\cline { 2 - 6 } & 25 & 0.6323 & 0.6362 & 0.7143 & 0.8045 \\
\cline { 2 - 6 } & 30 & 2.2377 & 2.2377 & 2.2396 & 2.2454 \\
\cline { 2 - 6 } & 35 & 5.0622 & 5.0567 & 4.9556 & 4.8561 \\
\cline { 2 - 6 } & 40 & 8.8177 & 8.8101 & 8.6749 & 8.5511 \\
\hline \multirow{5}{*}{$\mathbf{0 . 3 0}$} & 20 & 0.1998 & 0.2044 & 0.3052 & 0.4440 \\
\cline { 2 - 6 } & 25 & 1.0000 & 1.0062 & 1.1128 & 1.2367 \\
\cline { 2 - 6 } & 30 & 2.8063 & 2.8063 & 2.8095 & 2.8195 \\
\cline { 2 - 6 } & 35 & 5.6431 & 5.6363 & 5.5129 & 5.3926 \\
\cline { 2 - 6 } & 40 & 9.2761 & 9.2656 & 9.0786 & 8.9041 \\
\hline
\end{tabular}

The figure 1 shows that the put option price for $\beta=$ $0,1,1.95$ are approximately equal, and the graph overlapping to each other when the stock price decreases.

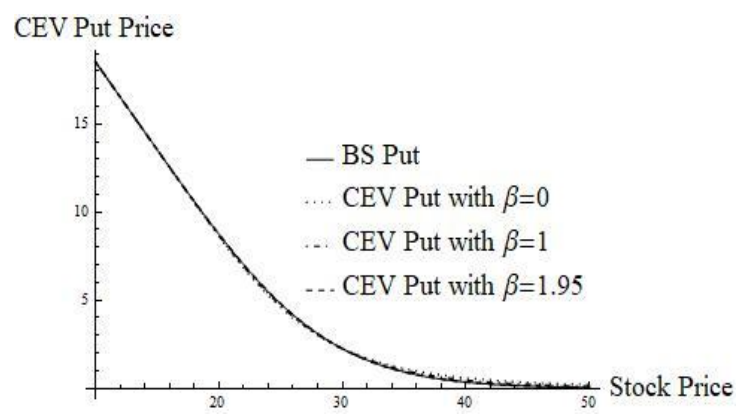

Fig. 1. Comparison of BS and CEV put option for $\beta=0, \beta=$ 1 , and $\beta=1.95$ with stock price at time $T=1$.

In particular, for strike price 30 the BS put price is 2.23768 and the CEV put price is 2.23769, 2.23958 and 2.24541 for $\beta=1.95, \beta=1.0$ and $\beta=0$ respectively. Table 1 shows that for ITM, when the strike price is 40 then the $\mathrm{CEV}$ put price is $8.81006,8.67486$ and 8.55106 for $\beta=1.95, \beta=1.0$ and $\beta=0$ respectively, where the BS put price is 8.81769 . For OTM, when the strike price is 
20 then the $\mathrm{CEV}$ put price is $0.0825025,0.135773$ and 0.214359 for $\beta=1.95, \beta=1.0$ and $\hat{\mathrm{a}}=0$ respectively, where the BS put price is 0.0802. The CEV model for put option gives consistantly under-price ITM and over-price OTM option.

\section{CEV Greeks vs. B-S Greeks}

Greeks provide important information about derivatives of options w.r.to different parameters, say, stock price, interest rate, volatility etc ${ }^{5}$.Each Greek has practical and theoretical importance and it measures a different dimension to the risk in a option position. Therefore, a trader's aim is to hedge it. Greeks like delta $(\partial / \partial S), \operatorname{gamma}\left(\partial^{2} / \partial S^{2}\right), \operatorname{vega}(\partial / \partial \sigma)$, $\operatorname{theta}(\partial / \partial t)$ and rho $(\partial / \partial t)$ are well documented in the literature in case of European option ${ }^{7}$. Now, we will discuss the Greeks for European put options under the CEV diffusion $\beta<2$. We have already discussed in section 2.2 the European put options formula for $\mathrm{CEV}$ diffusion, where

$$
\begin{aligned}
& 2 x=\frac{4(r-q) \exp ((r-q)(2-\beta) \tau)}{\delta^{2}(2-\beta)[\exp ((r-q)(2-\beta) \tau)-1]} S^{(2-\beta)} \\
& 2 y=\frac{4(r-q)}{\delta^{2}(2-\beta)[\exp ((r-q)(2-\beta) \tau)-1]} X^{(2-\beta)}
\end{aligned}
$$

To derive the Greeks under the CEV diffusion we can use some relations. Consider the relation $Q(w ; v, \lambda)=1-$ $F(w ; v, \lambda)$ we conclude that

$$
\begin{gathered}
\frac{\partial Q(w, v, \lambda)}{\partial w}=\frac{\partial[1-F(w ; v, \lambda)]}{\partial w}=-\frac{\partial F(w ; v, \lambda)]}{\partial w} \\
=-p(w ; v, \lambda) \\
\frac{\partial Q(w, v, \lambda)}{\partial \lambda}=\frac{\partial[1-F(w ; v, \lambda)]}{\partial \lambda}=-\frac{\partial F(w ; v, \lambda)]}{\partial \lambda} \\
=-p(w ; v+2, \lambda)
\end{gathered}
$$

where $p(w ; v, \lambda)$ is the pdf of a non-central chi-square distribution.

- To compute delta and gamma, we also need the auxiliary functions as below:

$$
\frac{\partial 2 x}{\partial S}=\frac{2 x(2-\beta)}{S} \text { and } \frac{\partial 2 y}{\partial S}=0
$$

Using these functions we can derive the delta of a put for $\beta<2$,

$$
\begin{aligned}
& \Delta_{p u t}=\frac{\partial p}{\partial S}=-\exp (-q \tau) \quad[1-Q(2 y ; 2+ \\
&\left.\left.\frac{2}{2-\beta}, 2 x\right)\right]+\frac{2 x(2-\beta)}{S}[S \\
&\left.-Q\left(2 y ; 2+\frac{2}{2-\beta}, 2 x\right)\right]+[S \exp (-q \tau) \\
& \quad\left[p\left(2 y ; 4+\frac{2}{2-\beta}, 2 x\right)\right. \\
&\left.\quad-X \exp (-r \tau) \quad p\left(2 x ; \frac{2}{2-\beta}, 2 y\right)\right]<0
\end{aligned}
$$

- To calculate gamma we also need to use the recurrence relations obtained by Cohen $^{7}$ :

$$
\begin{gathered}
\frac{\partial p(w ; v, \lambda)}{\partial \lambda}=\frac{1}{2}[-p(w ; v, \lambda)+p(w ; v+2, \lambda)] \\
\frac{\partial p(w ; v, \lambda)}{\partial w}=\frac{1}{2}[-p(w ; v, \lambda)+p(w ; v-2, \lambda)] \\
\left.p(w ; v-2, \lambda)=\frac{\lambda}{w} p(w ; v+2, \lambda)+\frac{v-2}{w} p(w ; v, \lambda)\right]
\end{gathered}
$$

Using these recurrence relations the put gamma for $\beta<2$,

$$
\begin{aligned}
& \Gamma_{p u t}=\frac{\partial^{2} c}{\partial S^{2}}= \frac{2 x(2-\beta)^{2}}{S} \exp (-q r) \\
& {\left[\left(\frac{3-\beta}{2-\beta}-x\right) p\left(2 y ; 4+\frac{2}{2-\beta}, 2 x\right)\right.} \\
&\left.+x P\left(2 y ; 6+\frac{2}{2-\beta}, 2 x\right)\right] \\
&+ \frac{2 x(2-\beta)^{2}}{S} \exp (-q r) \frac{X}{S} \\
&\left.-y p\left(2 x ; \frac{2}{2-\beta}, 2 y\right)\right]>0
\end{aligned}
$$

- $\quad$ The theta of put for $\beta<2$

$$
\begin{gathered}
\theta_{p u t}=\frac{\partial p}{\partial t}=-\frac{\partial p}{\partial \tau}=\operatorname{Xrexp}(-r \tau) \\
Q\left(2 x ; \frac{2}{2-\beta}, 2 y\right)-S q \exp (-q \tau) \\
{\left[1-Q\left(2 y ; 2+\frac{2}{2-\beta}, 2 x\right)\right]+} \\
\frac{2 x(r-q)(2-\beta)}{\exp ((r-q)(2-\beta) \tau)-1}[S q \exp (-q \tau) \\
p\left(2 y ; 4+\frac{2}{2-\beta}, 2 x\right)- \\
\left.X \exp (-r \tau) p\left(2 x ; \frac{2}{2-\beta}, 2 y\right)\right]
\end{gathered}
$$

- $\quad$ The Vega of put option for $\beta<2$,

$$
\begin{aligned}
& \text { Vega }=\frac{\partial c}{\partial \sigma}=\frac{\partial p}{\partial \sigma}=-\frac{4 x}{\sigma} \\
& {\left[\operatorname{Sexp}(-q \tau) \quad p\left(2 y ; 4+\frac{2}{2-\beta}, 2 x\right)\right.} \\
& \quad-X r \exp (-r \tau) \quad p\left(2 x ; \frac{2}{2-\beta}, 2 y\right)>0
\end{aligned}
$$


- $\quad$ The Rho of put for $\beta<2$,

$$
\begin{aligned}
\rho_{p u t}= & \frac{\partial c}{\partial r}=-X \tau \exp (-r \tau)-[1- \\
& \left.Q\left(2 x ; \frac{2}{2-\beta}, 2 y\right)\right]+2 x\left(\frac{1}{r-q}-\right. \\
& \left.\frac{(2-\beta) r}{\exp ((r-q)(2-\beta) \tau)-1}\right) \times[S \\
& \exp (-q \tau) p\left(2 y ; 4+\frac{2}{2-\beta}, 2 x\right) \\
& \left.-X \exp (-r \tau) \quad p\left(2 x ; \frac{2}{2-\beta}, 2 y\right)\right]<0
\end{aligned}
$$

To compute Greeks we use the same data set discussed in section 3. The calculated put option greeks under CEV diffusion and BS model are shown in the tables below:

Table 2. European Greeks put price with â $=0,0.5,1,1.5,1.95$ and for the strike price $X=$ 25, 30, 35 under the CEV model

\begin{tabular}{|l|l|l|l|l|l|l|}
\hline $\mathbf{X}$ & \multicolumn{1}{|c|}{$\boldsymbol{D}$} & Delta & Gamma & Vega & Theta & Rho \\
\hline \multirow{5}{*}{25} & 0 & -0.198 & 0.038 & 8.135 & -0.732 & -5.700 \\
\cline { 2 - 7 } & 0.5 & -0.183 & 0.036 & 7.792 & -0.698 & -5.52 \\
\cline { 2 - 7 } & 1 & -0.170 & 0.034 & 7.468 & -0.666 & -5.35 \\
\cline { 2 - 7 } & 1.5 & -0.158 & 0.032 & 7.160 & -0.636 & -5.18 \\
\cline { 2 - 7 } & 1.95 & -0.147 & 0.031 & 6.894 & -0.611 & -5.03 \\
\hline \multirow{5}{*}{30} & 0 & -0.421 & 0.053 & 11.44 & -0.759 & -13.4 \\
\cline { 2 - 7 } & 0.5 & -0.409 & 0.053 & 11.40 & -0.755 & -13.4 \\
\cline { 2 - 7 } & 1 & -0.397 & 0.052 & 11.37 & -0.751 & -13.4 \\
\cline { 2 - 7 } & 1.5 & -0.385 & 0.051 & 11.36 & -0.749 & -13.4 \\
\cline { 2 - 7 } & 1.95 & -0.374 & 0.051 & 11.35 & -0.748 & -13.4 \\
\hline \multirow{5}{*}{35} & 0 & -0.674 & 0.049 & 10.55 & -0.133 & -23.7 \\
\cline { 2 - 7 } & 0.5 & -0.660 & 0.05 & 10.76 & -0.162 & -23.7 \\
\cline { 2 - 7 } & 1 & -0.645 & 0.050 & 10.99 & -0.192 & -23.6 \\
\cline { 2 - 7 } & 1.5 & -0.630 & 0.051 & 11.22 & -0.224 & -23.6 \\
\hline & 1.95 & -0.616 & 0.051 & 11.44 & -0.255 & -23.5 \\
\hline
\end{tabular}

Table 3. European Greeks put price for the strike price $X=25,30,35$ under BS model

\begin{tabular}{|l|l|l|l|l|l|}
\hline $\mathbf{X}$ & Delta & Gamma & Vega & Theta & Rho \\
\hline 25 & -0.1459 & 0.0305 & 6.8654 & -0.6078 & -5.01 \\
\hline 30 & -0.3726 & 0.0505 & 11.353 & -0.7483 & -13.42 \\
\hline 35 & -0.6147 & 0.0510 & 11.470 & -0.2586 & -23.50 \\
\hline
\end{tabular}

Tables 2 and 3 show that when the strike price is 25 (ITM) then the CEV vega (put) price is 6.8944 , for $\beta=1.95$, where the BS vega (put) price is 6.86543 . When the strike price is 35 (OTM) the CEV vega (put) price is -23.5098, where the BS rho put price is -23.5033 . But ATM the BS vega gives the price 11.3526 and the CEV diffusion price for vega is 11.3527 when the strike price is 30 . Therefore we can say that the CEV vega gives the over price for ITM and under price for OTM options.

Figure 2 below represents the values of Greeks of put option for $\beta=0,1,1.95$ under CEV model and it's comparison to the values of Greeks under B-S model.

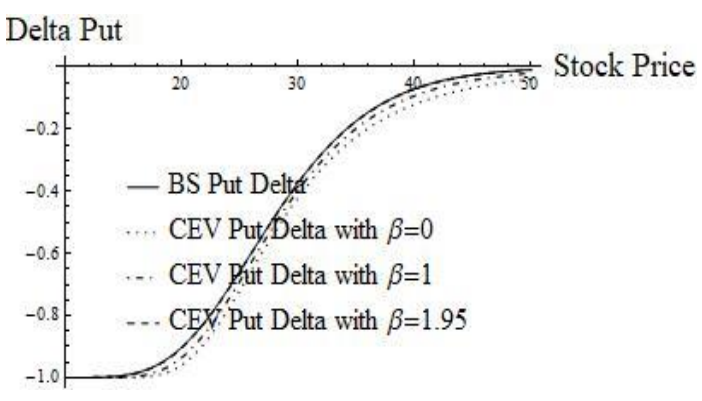

(a) BS vs. CEV Delta Put

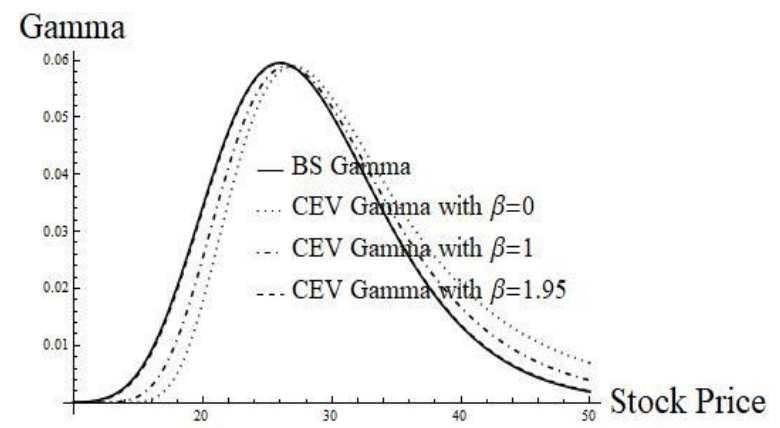

(b) BS vs. CEV Gamma Put

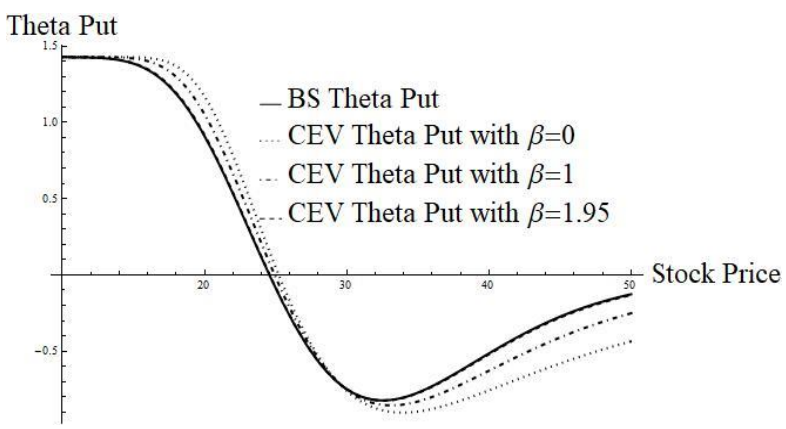

(c) BS vs. CEV Theta Put

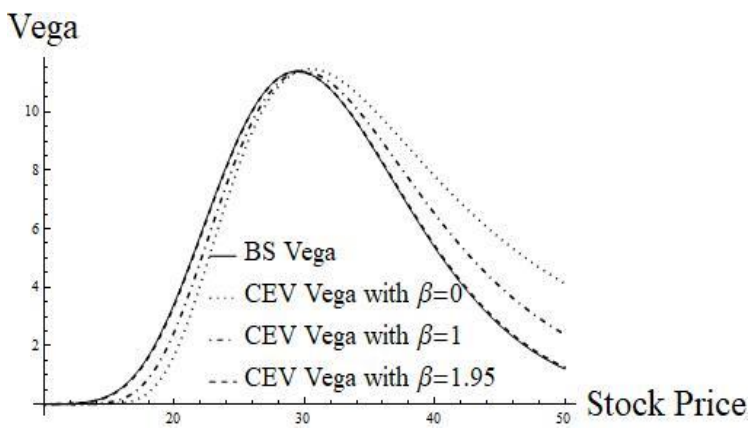

(d) BS vs. CEV Vega Put 


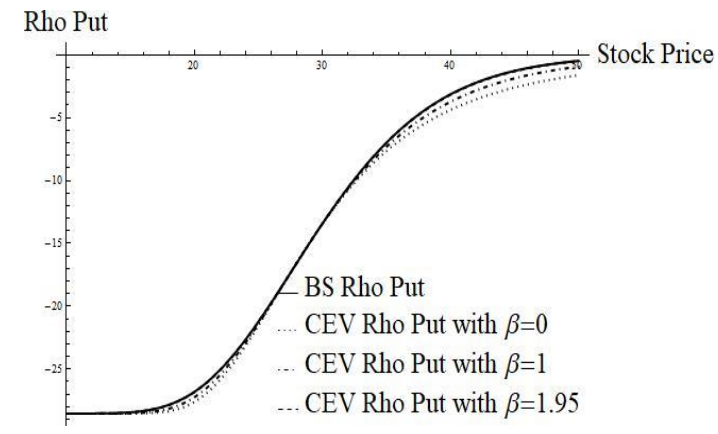

(e) BS vs. CEV Rho Put

Fig. 2. Variation of (a) delta (b) gamma (c) theta (d) vega and (e) rho of a put option under BS and CEV diffusion for $\beta=0,1,1.95$ with stock price.

Figure 3, 4 and 5 represents variation of some Greeks of a put option under BS and CEV diffusion for $\beta=0,1,1.95$ with time to maturity.

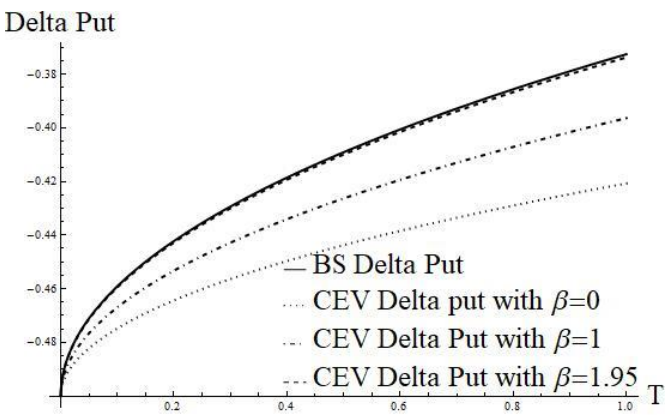

(a) Delta put (ATM)

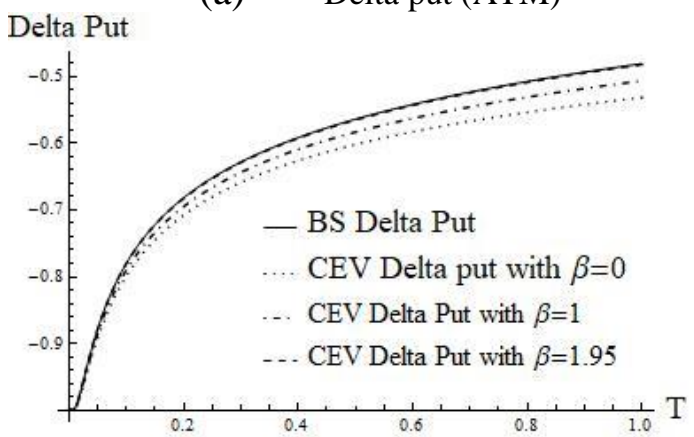

(b) Delta put (ITM)

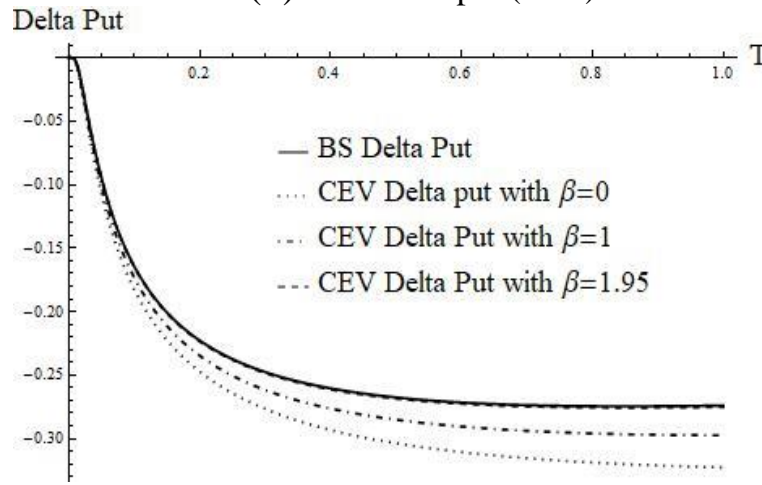

(c) Delta put (OTM)

Fig. 3. Variation of delta (a), (b), (c) of a put options under BS and CEV diffusion for $\beta=0,1,1.95$ with time to maturity

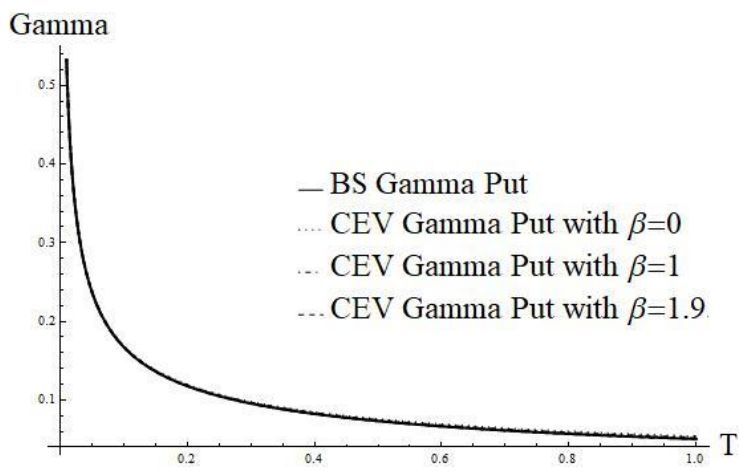

(a) Gamma put (ATM)

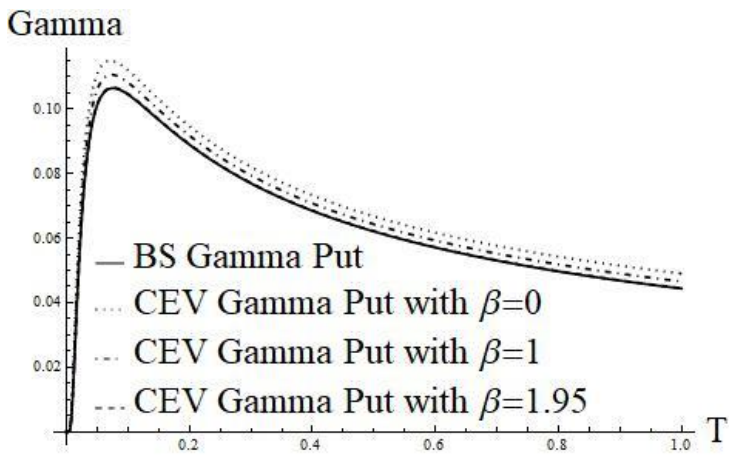

(b) Gamma put (ITM)

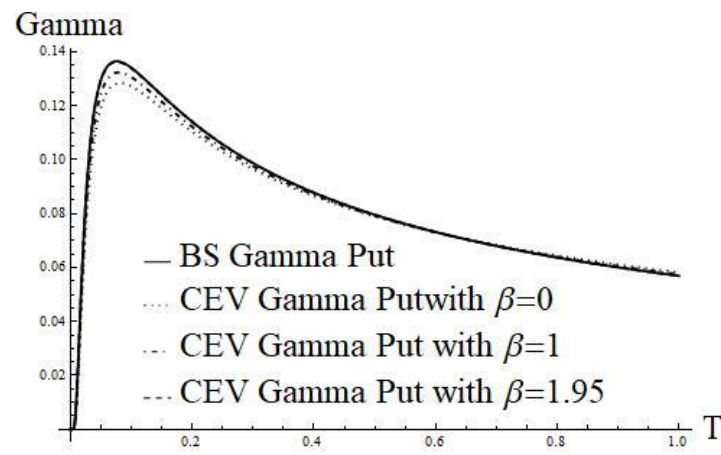

(c) Gamma put (OTM)

Fig. 4. Variation of gamma (d), (e) and (f) of a put options under BS and CEV diffusion for $\beta=0,1,1.95$ with time to maturity

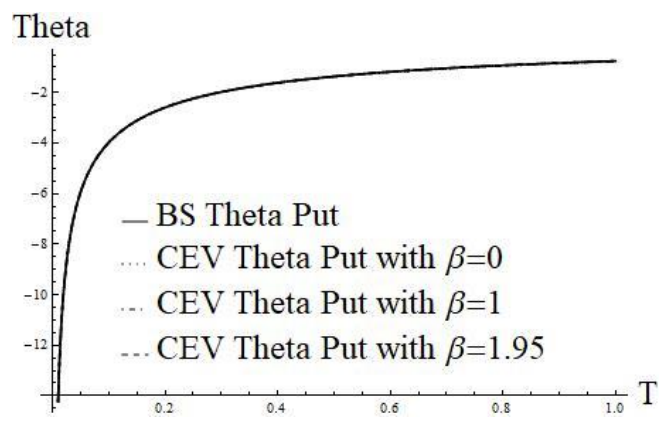

(a) Theta put (ATM) 


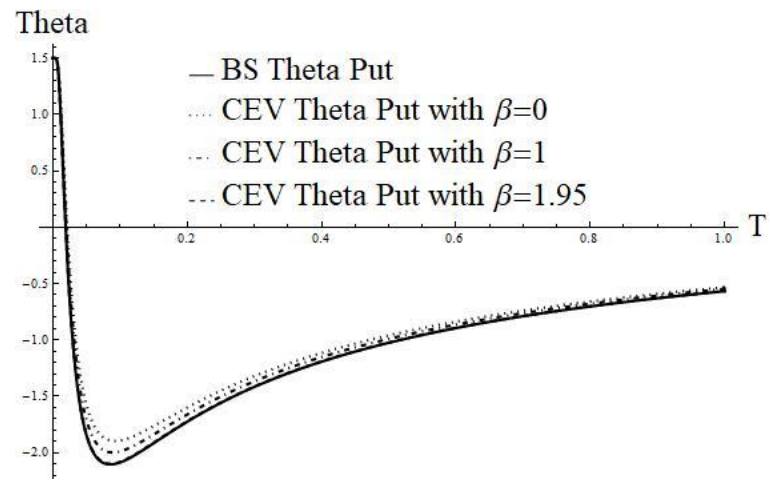

(b) Theta put (ITM)

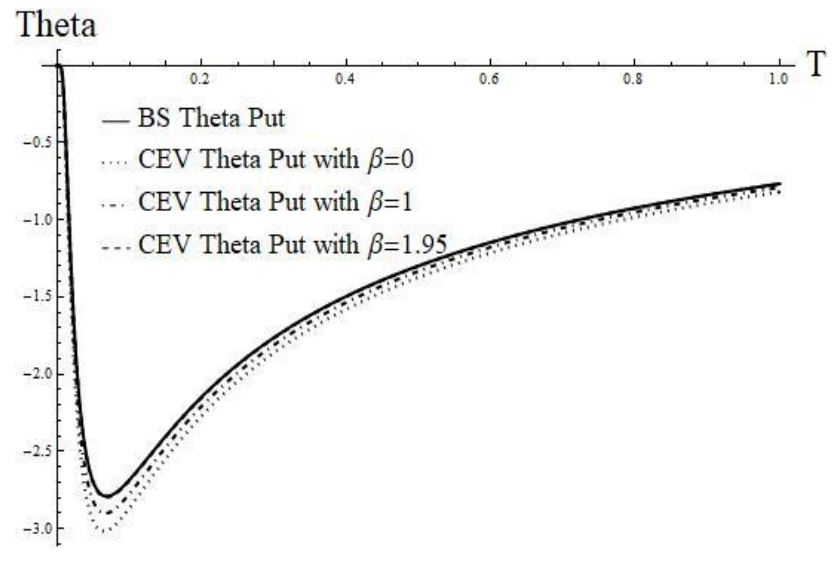

(c) Theta put (OTM)

Fig. 5. Variation of Theta (a), (b), (c) of put options under BS and CEV diffusion for $\beta=0,1,1.95$ withtime to maturity.

\section{Conclusion}

We may conclude that the volatility $(\sigma)$ increases as the stock price $(S)$ decreases and the BS model price, with the correct volatility $(\sigma)$, is greater than the market price for OTM options and less than the market price for ITM options. As long as the BS model has the correct volatility $(\sigma)$, it yields the market price for an (approximately) ATM option. Thus, in a constant elasticity of variance world, the CEV model yields prices that are better than BS prices. Because, firstly, the CEV adjusts the mispricing of OTM and ITM options and, secondly, it guesses changes in $\sigma$. The Greeks under the CEV model sometimes perform better or similar to that of the BS model for different values of $\beta$.

\section{References}

1. Black, F., and M. Scholes, 1973. The pricing of options and corporate liabilities, Journal of political economy, 81(3), 637-654.

2. Emanuel, D. C. and J. D.MacBeth, 1982. Further results on the constant elasticity of variance call option pricing model. Journal of Financial and Quantitative Analysis, 17(04), 533554 .

3. MacBeth, J. D., and L. J. Merville, 1980. Tests of the BlackScholes and Cox Call Option Valuation Models. The Journal of Finance, 35(2), 285-301.

4. Beckers, S. 1980. The constant elasticity of variance model and its implications for option pricing. The Journal of Finance, 35(3), 661-673.

5. Hull, John C., 2012. Options, futures, and other derivatives. 8th ed. Upper Saddle River, NJ: Prentice Hall.

6. Cox, J., 1975. Notes on option pricing I: Constant elasticity of variance diffusions. Unpublished note, Stanford University, Graduate School of Business.

7. Cohen, J. D., 1988. Non central chi-square: Some observations on recurrence. The American Statistician, 42(2), 120-122. 